This item was submitted to Loughborough's Research Repository by the author.

Items in Figshare are protected by copyright, with all rights reserved, unless otherwise indicated.

\title{
What came before the crash? An investigation through SHRP2 NDS data
}

PLEASE CITE THE PUBLISHED VERSION

https://doi.org/10.1016/j.ssci.2019.03.010

PUBLISHER

(c) Elsevier

VERSION

AM (Accepted Manuscript)

PUBLISHER STATEMENT

This paper was accepted for publication in the journal Safety Science and the definitive published version is available at https://doi.org/10.1016/j.ssci.2019.03.010

LICENCE

CC BY-NC-ND 4.0

\section{REPOSITORY RECORD}

Papazikou, Evita, Mohammed Quddus, Pete Thomas, and David Kidd. 2019. "What Came Before the Crash? an Investigation Through SHRP2 NDS Data". figshare. https://hdl.handle.net/2134/37686. 
1 What came before the crash?

2 An investigation through SHRP2 NDS data.

3

4 


\begin{abstract}
Investigating crash progression through naturalistic driving studies (NDS) could give valuable insights in crash causation analysis and thus, benefit crash prevention. This study utilises NDS data from the Strategic Highway Research Program 2 (SHRP2 NDS data) to look into the whole crash sequence, from a normal driving situation until a crash or a near-crash event. The objectives are to explore vehicle kinematics before the event, investigate the feasibility of crash risk indicators to detect the early stages of crash development and further examine the factors affecting Time To Collision (TTC) values during the crash sequence. An empirical approach and a multilevel mixed effects modelling technique were followed. The results reveal that longitudinal acceleration, lateral acceleration and yaw rate can be reliable indicators for detecting deviations from normal driving. Moreover, TTC values are affected by vehicle type, speed of the ego vehicle, longitudinal acceleration and time within the crash sequence. The model indicates a timestamp where a detectable reduction in TTC values occurs, which could be a first step towards more effective Advanced Driver Assistance Systems (ADAS) aiming to halt early deviations before they evolve to mishaps.
\end{abstract}

Keywords: Time To Collision (TTC); multilevel mixed effects modelling; crash sequence; SHRP2 NDS data; Naturalistic Driving Studies; safety indicators.

\title{
Highlights
}

- An empirical methodology to launch indicators for exploring pre-crash conditions regarding vehicle kinematics in NDS was developed.

- A unique process, to identify the critical time point where TTC values starting to drop during the event development, was employed based on multilevel modelling technique.

- The findings of this study could pave the way in the development of new ADAS, rendering them able to more effectively and timely detect, and stop an early deviation before it culminates in a crash. 


\section{INTRODUCTION}

One of the major challenges for the reduction or even elimination of crashes is the timely detection of critical and dangerous situations and then the development of appropriate interventions to prevent them (Lefèvre et al., 2014). Traditionally, crash causation analysis has relied mostly on crash statistics as the main data source. However, crashes are usually underreported and detailed information for the pre-crash traffic conditions, or for the behavioural aspects of road users is rarely available.

To address these issues, new trends in research on crashes involve Naturalistic Driving Studies (NDS). NDS are based on wide ranging collection of data regarding the driver, the vehicle, and the environment information in real world and these data sets could be valuable for the road safety analysis as they give the opportunity not only to measure and investigate the vehicle kinematics, but also to observe the driver behaviour, moments before the crash.

More specifically, Wu and Thor (2015) argue that it will be beneficial to use NDS data for crash sequence analysis by "quantifying and characterizing a crash surrogate during the progression of a crash". Therefore, there is the opportunity of measuring and predicting the probability of a scenario concluding in a crash. Towards this direction, surrogate safety measures, i.e., safety indicators have been employed to better understand the time dependent nature of relationships of crash causation factors (Laureshyn, Svensson, \& Hydén, 2010). Although there is a wide variety of safety indicators presented in the literature (Tarko et al., 2009), longitudinal and lateral acceleration, yaw rate and Time To Collision (TTC) are ubiquitous and have been widely used for crash proximity or occurrence detection. Improving the understanding between these indicators and the development of near crashes or crashes provides the opportunity for the development of new, more effective systems to support the driver in order to avoid crashes.

Detecting hazardous situations and investigating them in an effort to understand the evolution of the crash process would likely lead to a reduction of crashes. More particularly, a vehicle-based system that could automatically detect the deviation from normal driving by examining real-time kinematics data and prevent it in the first stage of the crash development process, before it culminates in a crash, could be fundamentally beneficial to road safety (Regan et al., 2009). This is challenging though, as fundamental research efforts are required to achieve an in-depth understanding of crash sequence.

Despite the existence of many studies estimating thresholds for Safety Critical Events (SCE) detection and unsafe or risky driving (Dingus et al., 2006, Hanowski et al., 2008, Olson et al., 2009, Benmimoun et al., 2011, Lerner et al., 2010, Blanco et al., 2008), the pathway of detecting the very initial deviation from normal driving during the crash or near crash development stages still remains a challenge (Jovanis et Wu, 2013). Additionally, many of the safety indicators in literature are study-specific or site-specific, with resulting restrictions of transferability and reproducibility (Aubin et al., 2014).

SHRP2 NDS provides the most comprehensive dataset with thousands of events (crashes and near crashes), vehicle and driver information available for investigation. Research questions that emerge are firstly, whether the data can be effectively used to examine what happens before the crash regarding vehicle kinematics and secondly, whether TTC is providing useful information about the pre-crash conditions. The objective of this study is, thus, to investigate through SHRP2 NDS data the whole crash sequence from a normal driving situation until a near-crash or a crash, examine how vehicle kinematics or driver related variables influence TTC values during the event progression and most importantly attempt to 
detect a timepoint where TTC values' pattern starts to reduce. In other words, the paper examines the feasibility of an indicator in early stages to be associated with crash risk and how far in advance before the event we can specify such a reliable indicator.

\section{PREVIOUS RESEARCH}

The purpose of this section is to review indicators of crash risk (e.g. acceleration, yaw rate, TTC) during pre-crash conditions with a special focus on data from NDS. The correlation between safety indicators associated with the development of a crash has been investigated with the purpose of understanding the crash mechanism, to avert or mitigate crash occurrences at various stages within the crash development process (Chapman, 1954; Evans, 2004). Tingvall et al. (2009) documented the so-called integrated safety chain where the stages before a critical event are described. Four stages are identified, starting from the deviation from normal driving, continuing with the emerging situation, then the critical situation and concluding to the stage where crash event is unavoidable. Figure 1 illustrates the crash development stages.

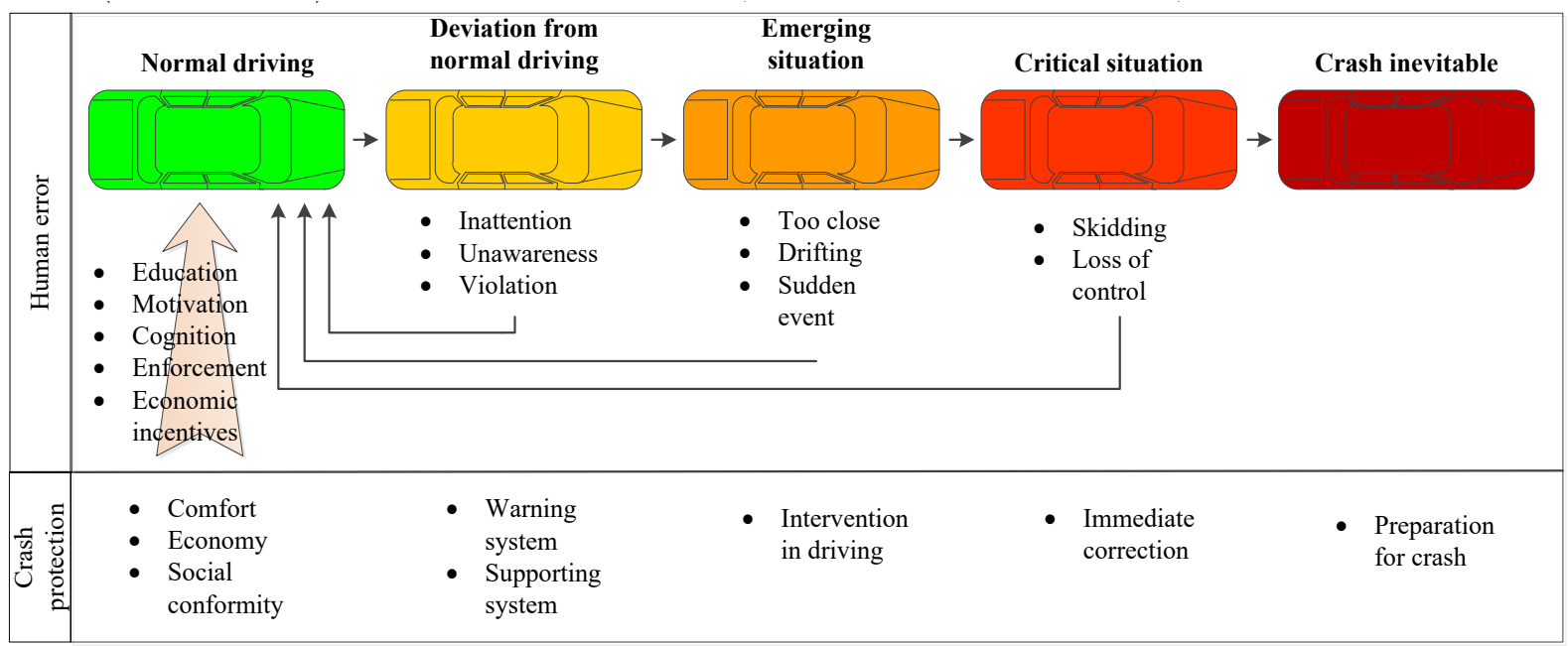

Figure 1 Stages of the crash development (adapted from (Tingvall et al., 2009))

\section{Safety indicators}

Through naturalistic driving datasets, it is possible to examine the different stages of the crash development and several safety indicators have been employed for this reason. In this section four of them will be briefly discussed according to the literature with focus on TTC which 
and set the basis for the modelling process of the TTC values during the crash or near crash development.

A range of different indicators have been used to measure the driving risks and possibly detect imminent hazardous situations. These primarily include: longitudinal and lateral acceleration, yaw rate and TTC. Longitudinal acceleration is commonly used to measure certain traffic conditions such as crashes and near-crashes in NDS or studies where kinematic vehicle data is gathered (McLaughlin et al., 2008; Nishimoto et al., 2001; Winsum \& Brouwer, 1997; Winsum \& Heino, 1996; Abdel-Aty et al., 2008) and it is thus considered to be a valid risk indicator. Moreover, longitudinal acceleration has been widely used for the investigation of braking behaviour or as indicator of driving performance. As a measure of braking, it is considered an indicator of a driver's perception of risk. More specifically, Bagdadi and Várhelyi (2013) analysed acceleration profiles and investigated the acceleration rate of change to distinguish critical and potential critical situations while Af Wåhlberg (2006) investigated the driver acceleration and deceleration behaviour and revealed that a combined celeration (overall mean speed change) measure constitutes a better predictor for crash involvement. Furthermore, it is found that drivers with a history of crashes indicate more abrupt braking, thus, the harsh braking responses could be an indication of crash proneness (Bagdadi \& Várhelyi, 2011). Acceleration is also an important variable in risk assessment physics-based motion models in the context of intelligent vehicles, allowing prediction of how a situation will evolve in the future (Lefèvre et al., 2014).

Yaw rate (degrees or radian/sec), is the instantaneous angular velocity of the vehicle rotation, or rate of change of the heading angle. It is an important measure that depicts the lateral change of the vehicle's direction and it is widely used as an indicator of vehicle lateral motion. For example, previous studies have utilised it for control algorithms and lane change models, (e.g. Park et al, 2015) or to examine the steering behaviour in collision avoidance and lateral movements (e.g. Wu et al, 2017). Additionally, Sudweeks (2015) used a functional yaw rate classifier which reduced the number of false triggers $42 \%$ relatively to the $100-C a r$ NDS (Dingus et al., 2006) and maintained the majority of safety relevant events (92\% of crashes, $81 \%$ of near-crashes).

Finally, indicators based on lateral acceleration have been utilised for lane change detection (e.g. Farid et al., 2006) and lateral vehicle kinematics measurements (Dingus et al., 2006).

All the above, (and TTC that will be discussed below), have been employed to better understand the mechanism of crash occurrence and have been also utilised in various studies as post-hoc triggers for the detection of SCE from NDS (Dingus et al., 2006; Pilgerstorfer et al., 2012; Hanowski et al., 2008; Olson et al., 2009; Benmimoun et al., 2011; Lerner et al., 2010; Blanco et al., 2008; McGehee et al., 2007; Hankey et al., 2016). While the vehicle remains under the control of the driver both longitudinal acceleration and yaw rate are considered to be direct measures of driver behaviour in response to a driving situation.

\section{Time-to-collision}

TTC offers a time dimension to the crash risk, it relies on motion prediction method and is continuously derived (typically from a radar). It is widely considered in the literature as a potential surrogate of conflicts and it is typically suggested as a trigger for collision avoidance systems. TTC's high transferability helps to that direction as it can be present in every driving scenario. In this study, as the development of an event is studied, the focus is on TTC as it can be calculated for any moment during the event sequence and it has been associated with 
driver reaction (Lee, 1976, Van Winsum and Heino, 1996, Van Winsum and Brouwer, 1997, Van Winsum, 1999). Last but not least, TTC stands out for its suitability to assess safety critical probabilities.

The original definition of TTC was devised by Hayward in 1972 and it is one of the most widely-used indicators of traffic safety (Laureshyn et al., 2010). When there is a collision course, TTC is the time that remains before the collision takes place. TTC values then become finite and decline with time unless a vehicle changes its heading or velocity. Therefore, minimum TTC during the conflict is the critical measurement to assess conflict severity.

TTC is a continuous variable, it can be calculated for any moment as long as the road users are on the collision course and it has been applied in several studies (e.g. Fancher et.al.,1998; Van Arem \& De Vos, 1997) to pinpoint traffic safety impacts (Archer, 2001). Moreover, Ward et al. (2015) calculated TTC for two dimensions extending the applicability of the safety indicator to more traffic scenarios.

Studies have used the recorded minimum value of TTC of relevant events, the value when evasive action began or alternatively the duration of the defined TTC event. There is still no general agreement between researchers regarding the critical value of TTC; nevertheless, for safety studies this value is proposed to be greater than 1.5 second and less than 5 seconds (Martens \& Brouwer, 2011). Hayward (1972) suggested that 4 seconds is a critical value of TTC below which, there is a crash prone situation, while Brown et al. (2001) identified a TTC threshold of 3 seconds.

Raw TTC measure cannot be considered as valid in all critical situations. TTC value is calculated from distance and speed, but can be ambiguous as two cars travelling at a speed of $20 \mathrm{~km} / \mathrm{h}$ and $160 \mathrm{~km} / \mathrm{h}$ respectively, could present the same TTC value. To discriminate between these unequal conditions, TTC can be used in combination with other measures e.g. absolute value of speed at defined TTC event or braking rate (Niezgoda et al., 2012).

Lee (1976) suggested the use of TTC rather than speed, distance or acceleration/deceleration for a controlling braking strategy. Farah et al. (2017) used TTC as a surrogate safety measure to investigate head-on collisions with the opposite vehicles during an overtaking manoeuvre on two-lane rural highways. Similarly, Hegeman (2008) and ShariatMohaymany (2011) have used TTC as a measure of crash risk during overtaking manoeuvres.

TTC is considered to be a significant factor for judging the moment to start braking, and the role of TTC information in the control of braking has been investigated (Van Winsum \& Heino, 1996). Recently, Lee et al (2016) introduced an advanced braking algorithm using new longitudinal safety index based on TTC and warning index while Li et al. (2016) proposed a collision risk algorithm for connected environment based on real-time TTC values.

Finally, several studies examined the factors affecting TTC. Farah et al. (2009) with the employment of a Tobit regression model investigated minimum TTC and proved that not only geometric design of road, but also traffic and driver characteristics explanatory variables present a significant effect on minimum TTC. Similarly, Van Winsum et al. (1997), (1999) considered both kinematic and human factors characteristics for a car following model based on TTC and the states of the following vehicles. Furthermore, with driving experiments, Hamdar et al. (2016) confirmed the real-world observations that roadway (lane width, shoulder width, median existence, median type, horizontal and vertical curves) and weather associated factors (foggy weather, icy and wet road surface conditions) influence TTC. Moreover, it was understandable that although different environmental conditions affect general driving behaviour, the magnitude of a change of normal driving behaviour depends on individual drivers. It is also important, therefore, to examine the effect of demographic characteristics. 
In this paper SHRP2 NDS data is utilised to investigate the factors affecting TTC values during event sequence and especially the change of TTC values over time with the objective to identify the timepoint where the first deviation from normal driving happens. According to the above literature, the TTC values could be modelled as follows:

\section{$T T C=f(r o a d$ characteristics, traffic conditions, vehicle type \& kinematics, driver behaviour)}

In the methodology and the result section, details about the exact modelling process and the final explanatory variable choice for the model formulation will be adequately justified.

\section{DATA AND MODELLING METHODOLOGY}

Examining NDS data is one of the best ways to investigate the crash sequence. For this purpose, comprehensive NDS data collected as part of SHRP2 were obtained through Virginia Tech Transportation Institute (VTTI).

2.5 minutes of trip-based time series data before the events (crashes and near crashes) and the corresponding driver and event data were requested. This came as a methodological decision as not only it would render the extraction of baseline events possible but also, it would give the opportunity to investigate the whole crash or near crash sequence from normal driving until the event.

SHRP2 data were obtained from VTTI in the form of four datasets: (i) Time series data, (ii) Demographic questionnaires of the drivers, (iii) Event detailed data and (iv) Event ID data key.

Once the SHRP2 NDS data were received, several steps were carried out to prepare the data for modelling. A deeper understanding of the data was also essential as significant decisions were made in order to create a suitable dataset for the analysis.

The first step was to combine the events in a dataset. Secondly, the data was checked and cleaned of inconsistences, errors and other problematic observations. In the process to identify variables that are going to be useful for the analysis, it was noted that TTC is not provided as a variable in the SHRP2 NDS data but could be calculated from other available variables. That, however, was not a trivial task. More specifically, the sensor platform within an ego-vehicle is capable of simultaneously tracking up to eight different targets that the egovehicle encounters within its radar field of view (range $\sim 200 \mathrm{~m}$ ). Each of the targets is individually tracked as Track 0 through 7 . With the raw radar variables, these objects can sometimes switch across different tracks making the calculation of TTC difficult. To resolve this, a post-processing method was developed by VTTI in order to ensure that that the same target is being tracked consistently. This is to identify cases where the target vehicle is moving to different lanes while in front of the ego-vehicle or if another new vehicle comes into the radar field of view. These post-processed range and relative velocity were used in this study to calculate TTC for which the following process is developed and adopted:

1. Identify whether the ego-vehicle and a target vehicle are stationary;

2. Determine the lead target vehicle from the distances between target vehicles and the front bumper of the ego-vehicle, projected onto the x-axis (longitudinal) of ego-vehicle. The target vehicle with the smallest distance was identified as the lead vehicle; 
3. Identify if the lead vehicle is in the same lane of the ego-vehicle. The relevant variable (i.e. Lane in the NDS data) has five options coded as follows:

2: there exists at least one lane to the right of the ego-vehicle that separates the ego-vehicle from the target;

1: the target is in the adjacent lane to the right of the ego-vehicle vehicle;

0 : the target is in the same lane as the ego-vehicle;

-1 : the target is in the adjacent lane to the left of the ego-vehicle vehicle;

-2 : there exists at least one lane to the left of the ego-vehicle that separates the ego-vehicle from the target.

It should be noted that oncoming targets are not classified into lanes and these targets were given null values for "Lane".

Several algorithms were investigated for calculating TTC, from a very restricted (i.e. considering only vehicles in the same lane with the ego-vehicle or the same direction or both) to a more general one, but finally the most appropriate was chosen after investigation of the relevant TTC values and the relation with the time headway and the missing values of the dataset. The following equation is employed in calculating TTC:

$$
\mathrm{TTC}=\frac{d}{|\Delta v|}, \quad \Delta \mathrm{u}<0
$$

In which: TTC is the TTC between the ego-vehicle and the closest lead target vehicle, $d$ is the distance (in $\mathrm{m}$ ) between the rear bumper of the lead target vehicle and the front bumper of the ego-vehicle, projected onto the x-axis (longitudinal) of ego-vehicle and $\Delta v$ is the $x$-axis (longitudinal) component of range rate (relative velocity) between the lead target and the ego-vehicle (in $\mathrm{m} / \mathrm{sec}$ ). $\Delta v$ can be directly obtained from the data. Cases where the egovehicle and a target vehicle are stationary are excluded.

For each of the crash and near-crash events, the sample contains 150 seconds of time-series data from the radar of the ego-vehicle. Since the sampling frequency of the radar is $10 \mathrm{~Hz}$, we have a total of 1,500 epochs just before the crash/near-crash event. However, it was not possible to calculate the TTC values for all 1,500 epochs sequentially. For instance, if there are no lead vehicles in front of the ego-vehicle, TTC cannot be calculated and if the speed of the lead vehicle is higher than the speed of the ego-vehicle then the TTC also cannot be calculated as this does not satisfy equation (1). This means that many crash and nearcrash events were excluded. More specifically, only the events with TTC values available for at least 1100 epochs out of 1,500 were considered. The time series data then was matched with driver and event information, producing the final dataset.

Cleaning, manipulating and transforming the data were undertaken but practical difficulties occurred due to the structure, the complexity, the size and the continuous nature of the data. Initially the dataset included 3,604 events with 989 drivers involved in all crash or near-crash events according to the SHRP2 threshold specifications. As discussed above, TTC could not be calculated for all epochs. Therefore, the final dataset only contains 127 crash events and 647 near-crash events with 553 different drivers. With the resulting driving data sampled at $10 \mathrm{~Hz}$, over 1 million data points were available for analysis. 
This study examines how the safety surrogate measures (TTC, longitudinal deceleration, longitudinal acceleration, lateral acceleration, yaw rate) for 127 crash and 647 near-crash events change with respect to the time progression from epoch 1 (normal condition) to epoch 1,500 (crash or near-crash condition). For instance, for the case of crash events, there are a total of 127 TTC values for each of the 1,500. In order to see how these 127 TTC values (from 127 events) changes from one epoch to the next, percentiles are calculated. Data were restructured in columns each containing all the values of each timepoint for all the events (e.g. 1st observation of the first event, 1st observation of the second event, ...1st observation of last event) and the fifth percentiles of each timepoint of all the events sequence, were calculated. Since low values of TTC, deceleration, negative lateral acceleration and negative yaw rate imply unsafe conditions, the left-hand side of their distributions is of interest. Therefore, calculating a $5^{\text {th }}$ percentile value is more logical than that of a $95^{\text {th }}$ percentile value. For instance, the $95^{\text {th }}$ percentile value of 127 TTC values is 168 seconds at epoch 1,500 whereas this is 0.8 seconds for the $5^{\text {th }}$ percentile value. This means that only $5 \%$ of the 127 observations have TTC values less than 0.8 seconds. Figure 2 illustrates the process that was followed to generate the $5^{\text {th }}$ percentile indicators for the crash relevant data. The procedure was the same for near-crash data and it was applied to TTC, deceleration, negative lateral acceleration and negative yaw rate. Lateral acceleration and yaw rate entire distributions were symmetrical, therefore only the negative values were chosen for analysis to comply with the choice of the $5^{\text {th }}$ percentile explained above.

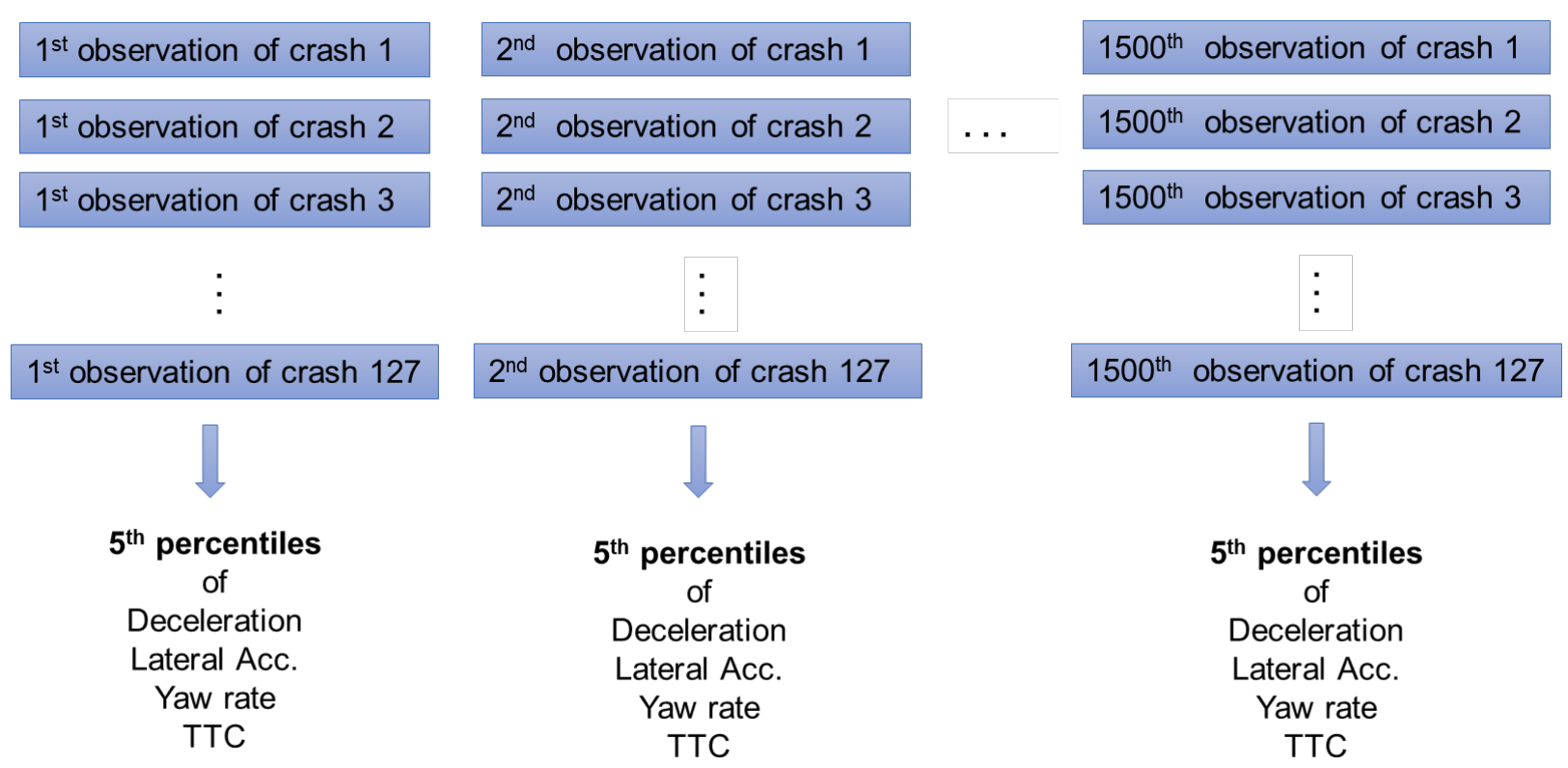


Analysis of these indicators could give a picture of the onset of the deviation in the course of the crash or near crash development. It is also worthwhile to examine the possible differences when the event severity changes from near crash to crash.

\section{Statistical modelling}

The literature reviewed in the previous section revealed factors that affect TTC values. A statistical model can examine how some of these factors are related to TTC within the crash sequence and whether there is a time point where a change in TTC values can be usefully detected. Considering the structure of the data, Hierarchical Linear Modelling (or multilevel mixed effects linear regression modelling) has been chosen for this study.

More specifically, three types of factors are considered: (1) driver factors (e.g. age, gender, miles driven previous year), (2) factors relating to the vehicle and vehicle kinematics (e.g. vehicle type, speed, yaw rate, lateral and longitudinal acceleration) and (3) factors related to the time within the event sequence (e.g. System timestamp, System timestamp squared). System timestamp variable represents the time to a crash or near-crash, therefore in this case of 2.5 minutes of data before the event and at $10 \mathrm{~Hz}$ frequency, timestamp varies from 1 (150 seconds before the event) to 1500 (when the event occurs). The quadratic relationship with TTC constitutes an assumption.

Since each driver had one or two trips and each trip had multiple observations (1500), TTC values during the event progression can be modelled using two level analyses i.e. the trip level and the observation level. More thoroughly, at the highest level of the hierarchy (level2) are vehicle type variable and driver related variables such as gender and age while in the lowest level (level-1) are vehicle kinematics and time variables such as speed, yaw rate, accelerations and system time stamp. Level 1 variables, observations, are impacted by level 2 variables, the trips. More specifically, the observations are nested within each trip-file in the dataset. The outcome variable is measured at level 1 as Hierarchical Linear Modelling (HLM) requires. The analysis aims to relate the driver gender and age, vehicle type, longitudinal and lateral acceleration, yaw rate, speed and time to TTC in the event sequence development. The model intends to detect when TTC values start to deviate from the norm to provide insights for potential detection of a hazardous situation.

Observations of vehicle kinematics from the same driver may have common characteristics (i.e. within-cluster correlation) or there might be a variation between the observations from different vehicle types (i.e. between-cluster variation). The use of a two level mixed effects linear regression model is suitable as it allows for dependency of the observations coming from the same driver and within the same trip and examines the variation of observations for different drivers and different trips by the same drivers. Furthermore, it deals with the issue of consistency since not all the drivers have executed multiple trips. A two-level mixed-effects linear regression model can be developed for a single explanatory variable $(x)$ as (Woltman et al., 2012):

Observations-level (level 1):

$Y_{i j}=\beta_{0 j}+\beta_{1 j} x_{i j}+e_{i j}$

Trip-file level (level 2):

$\beta_{0 j}=\gamma_{00}+\gamma_{01} w_{j}+u_{0 j} ; \quad \beta_{1 j}=\gamma_{10}+\gamma_{11} w_{j}+u_{1 j} ;$ 
The composite equation can be expressed as:

$Y_{i j k}=\gamma_{00}+\gamma_{10} x_{i j}+u_{1 j} x_{i j}+\gamma_{11} w_{j} x_{i j}+\gamma_{01} w_{j}+u_{0 j}+e_{i j}$

In which $Y_{i j}$ is the TTC values for ith level-1 observation nested within jth level-2 tripfile, $x_{i j}$ is the value for the level-1 predictor, $\beta_{0 j}$ is the intercept for the jth trip-file, $\beta_{1 j}$ is the regression coefficient (slope) associated with the $x_{i j}$ for the jth trip-file, $e_{i j}$ is the random error associated with the ith observation (level-1) nested within the $j$ th trip-file (level-2), $w_{j}$ is the value on the level-2 predictor, $\gamma_{00}$ is the overall mean intercept adjusted for $w, \gamma_{10}$ is the overall mean intercept adjusted for $\mathrm{w}, \gamma_{01}$ is the regression coefficient associated with $w$ relative to level-1 intercept, $\gamma_{11}$ is the regression coefficient associated with $w$ relative to level-1 slope, $u_{o j}$ is the random effects of the jth trip-file adjusted for $w$ on the intercept, $u_{1 j}$ is the random effects of the jth trip-file adjusted for $w$ on the slope. Subscript i refers to individual case therefore it can be 1 to 1500 (1500 observations per trip) while subscript $\mathrm{j}$ refers to the group and, in this study, can take values from 1 to 774 (774 trips).

All random components are assumed to follow a normal distribution with a mean of zero and a constant standard deviation. Equation (4) represents a two-level mixed-effects linear regression model for a single explanatory variable but this can be similarly extended for multiple explanatory variables. This model can be estimated using the restricted maximum likelihood (REML) estimation method.

\section{RESULTS}

This section consists of two parts. Firstly, the distributions of the $5^{\text {th }}$ percentile indicators, discussed in the previous section, are introduced, to show the change in vehicle kinematics leading to an event and subsequently, the multilevel modelling results regarding factors affecting TTC are presented.

\section{Exploratory analysis of vehicle kinematics before a crash or near crash}

Figures 3, 5, 7 \& 9 represent the crash sequence from normal driving until the crash events (127) and near crash events (647) for a range of safety indicators related to vehicle kinematics, namely, longitudinal deceleration, lateral acceleration (left side indicating negative values), yaw rate (negative values) and TTC. It was not possible to calculate the $5^{\text {th }}$ percentile value of longitudinal acceleration due to the very low sample size, especially for the crash events. For all the four variables, vehicle kinematics data related to near crash events differ from the corresponding data for the crash events (Mann-Whitney U test at the 95\% confidence level). The distributions and the corresponding descriptive statistics of these four indicators are also presented below (see Fig. 4, Fig. 6, Fig. 8, Fig. 10 and Table 1, Table 2, Table 3, Table 4) to indicate that surrogate safety measures vary by event severity. During the whole crash development, the magnitude of the variation of the indicator values for crashes is greater than this of near-crashes, but the most significant element is that the deceleration, lateral acceleration and yaw rate indicator values for crash events tend to reduce sharply approximately 10-20 seconds before the event indicating a clear deviation from the baseline driving. However, this is not the case with TTC. 


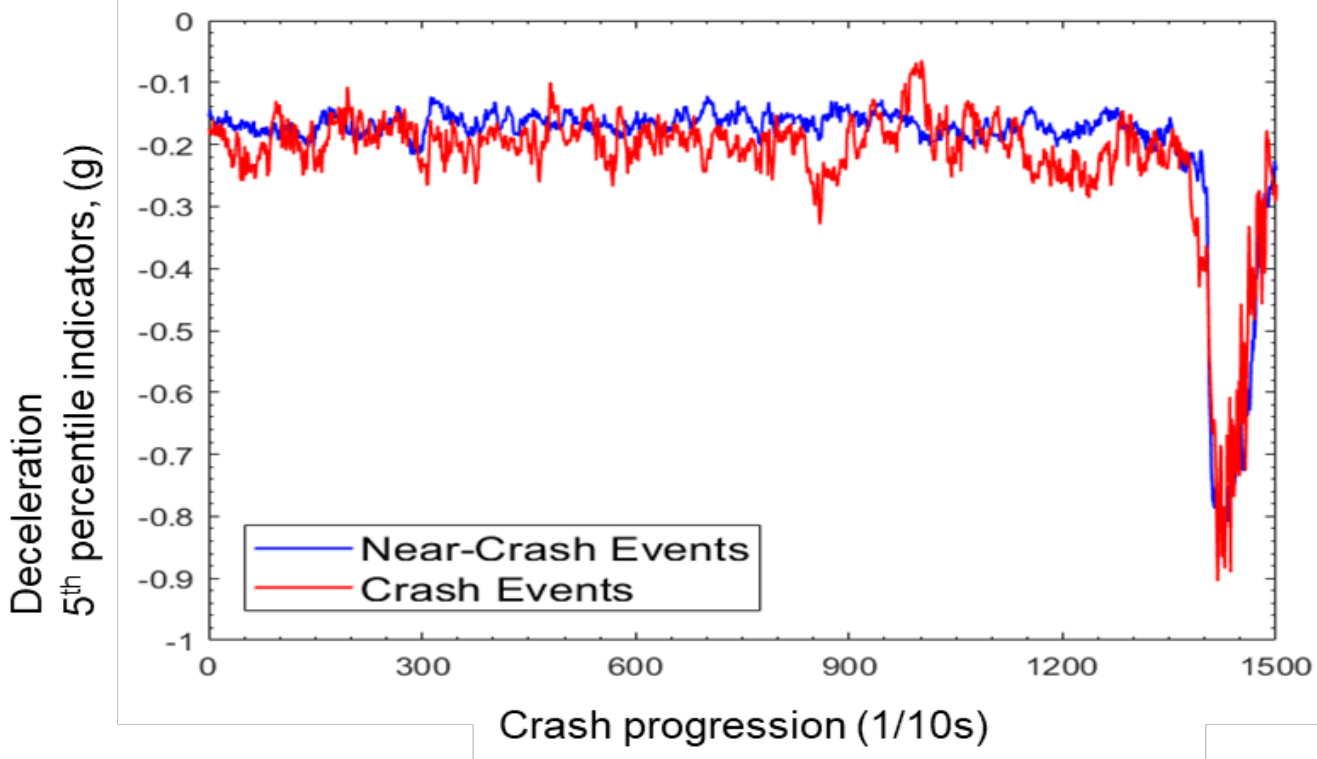

Figure 3 Deceleration 5th percentile indicators during crash \& near-crash development

4 Table 1 Descriptive statistics of Deceleration 5th percentile indicators

\begin{tabular}{lll}
\hline Deceleration $\mathbf{( g )}$ & Crashes (127) & Near Crashes (647) \\
\hline Mean & -.22 & -.196 \\
Median & -.198 & -.169 \\
Minimum & -.905 & -.817 \\
Maximum & -.064 & -.121 \\
\hline
\end{tabular}

5
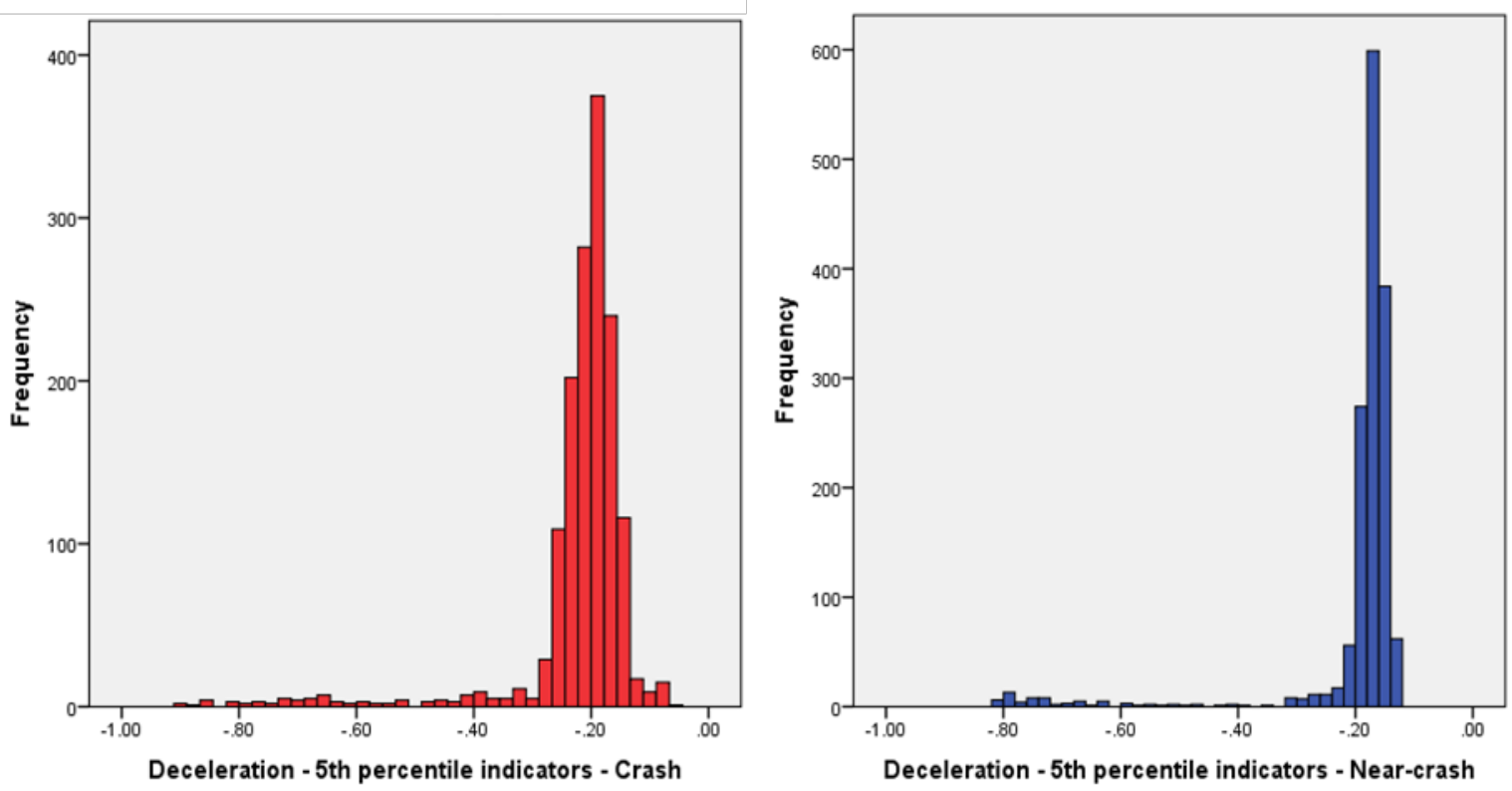

Figure 4 Distributions of Deceleration 5th percentile indicators for crash \& near crash events 


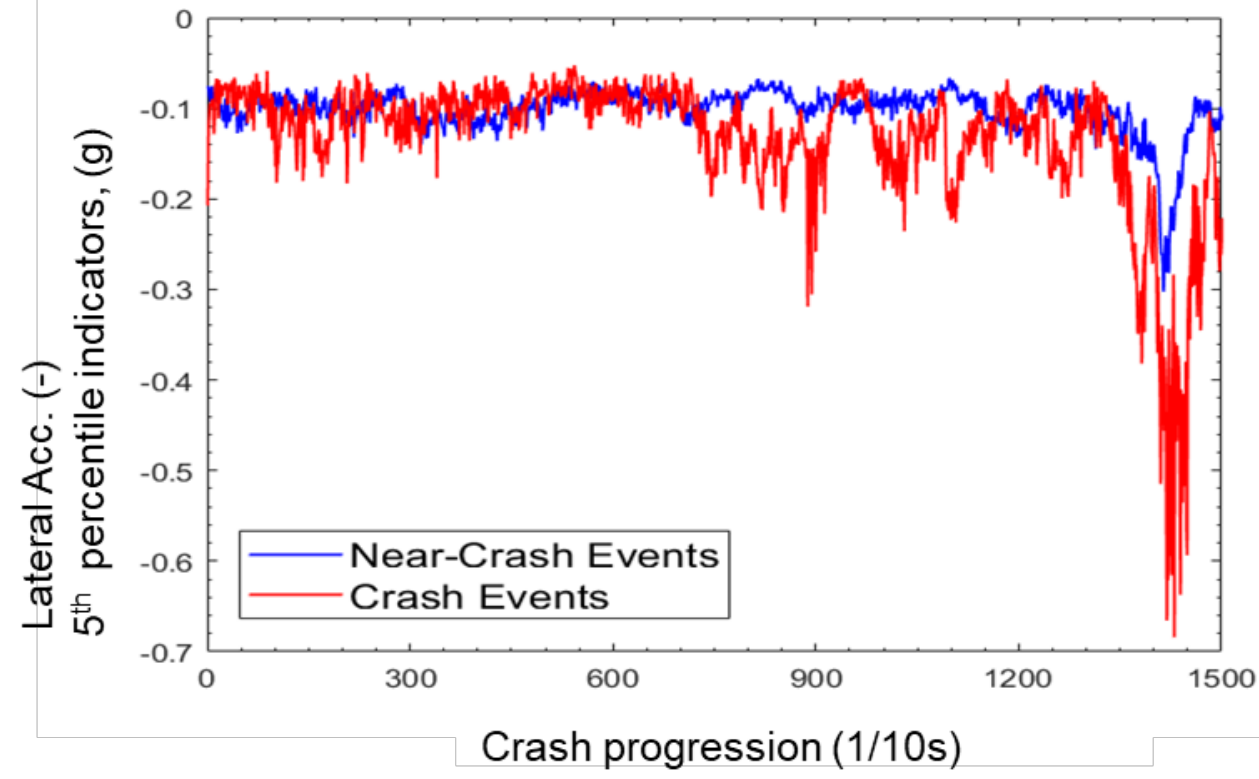

Figure 5 Lateral Acceleration (-) 5th percentile indicators during crash \& near-crash development

4

$5 \quad$ Table 2 Descriptive statistics of Lateral acceleration (-) 5th percentile indicators

\begin{tabular}{lll}
\hline Lateral Acc. (-), (g) & Crashes (127) & Near Crashes (647) \\
\hline Mean & -0.132 & -0.102 \\
Median & -0.112 & -0.096 \\
Minimum & -0.684 & -0.303 \\
Maximum & -0.052 & -0.067 \\
\hline
\end{tabular}

6

7
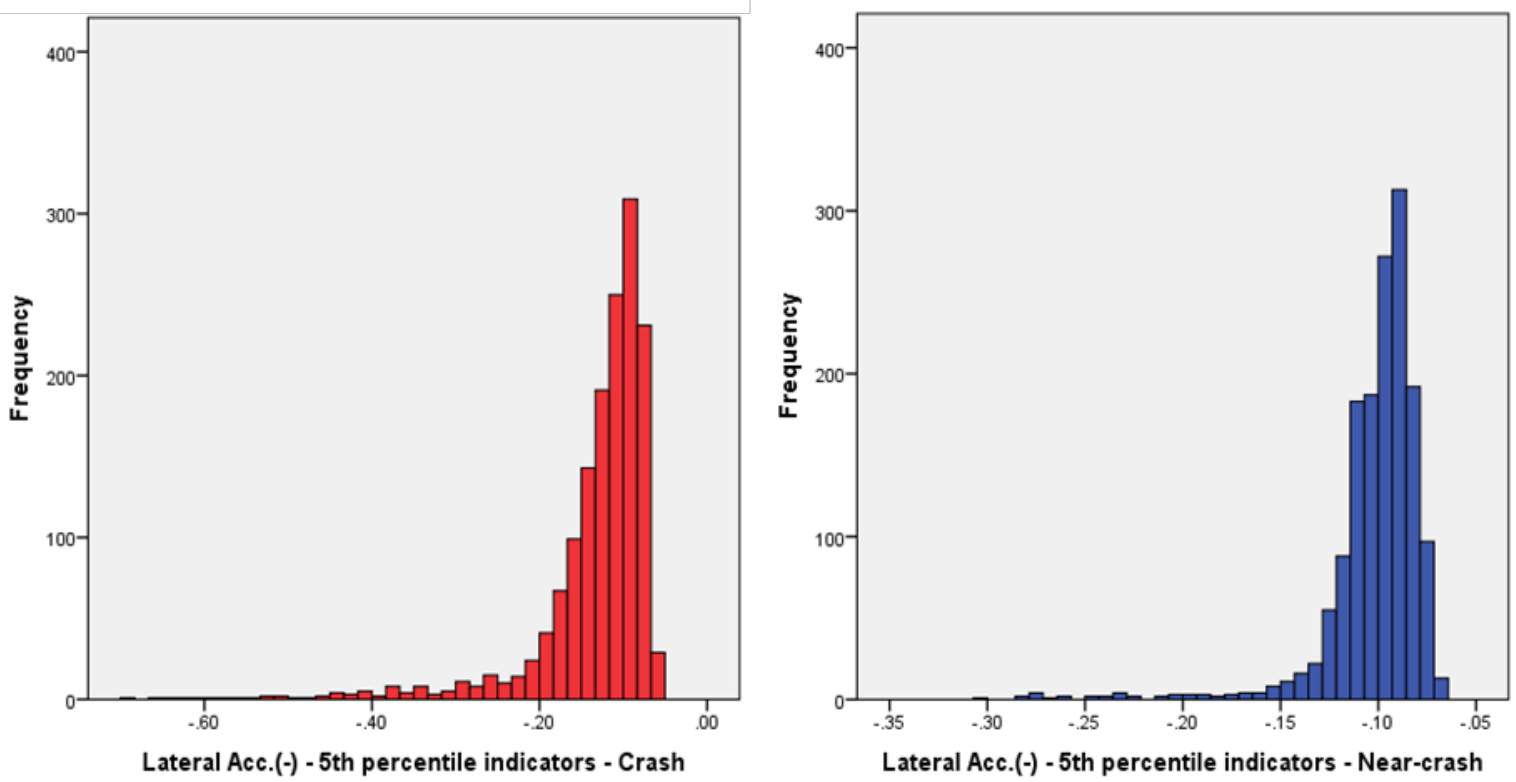

Figure 6 Distributions of Lateral Acceleration (-) 5th percentile indicators for crash \& near crash events 


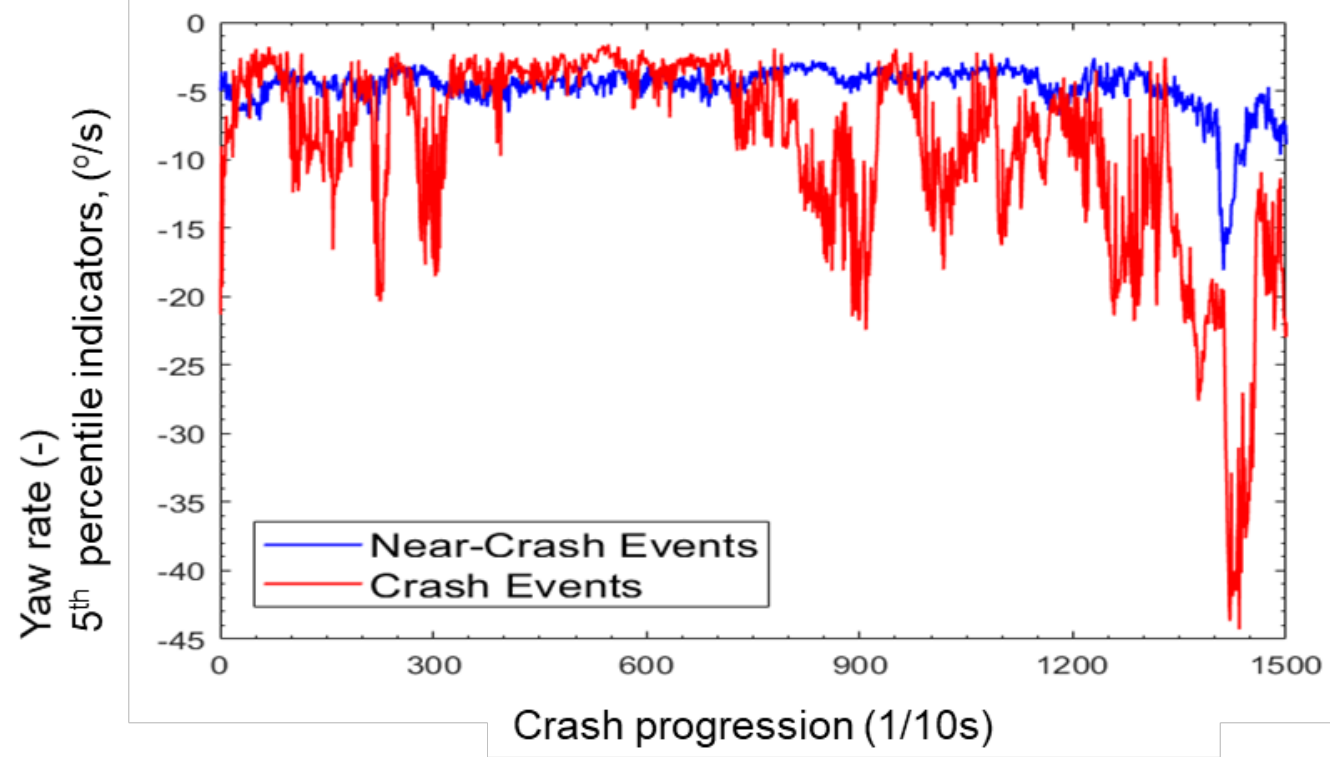

Figure 7 Yaw rate (-) 5th percentile indicators during crash \& near-crash development

4 Table 3 Descriptive statistics of Yaw rate (-) 5th percentile indicators

\begin{tabular}{lll}
\hline Yaw rate. (-), (\%) & Crashes (127) & Near Crashes (647) \\
\hline Mean & -9.140 & -4.751 \\
Median & -7.057 & -4.39 \\
Minimum & -44.292 & -18.097 \\
Maximum & -1.626 & -2.602 \\
\hline
\end{tabular}

5
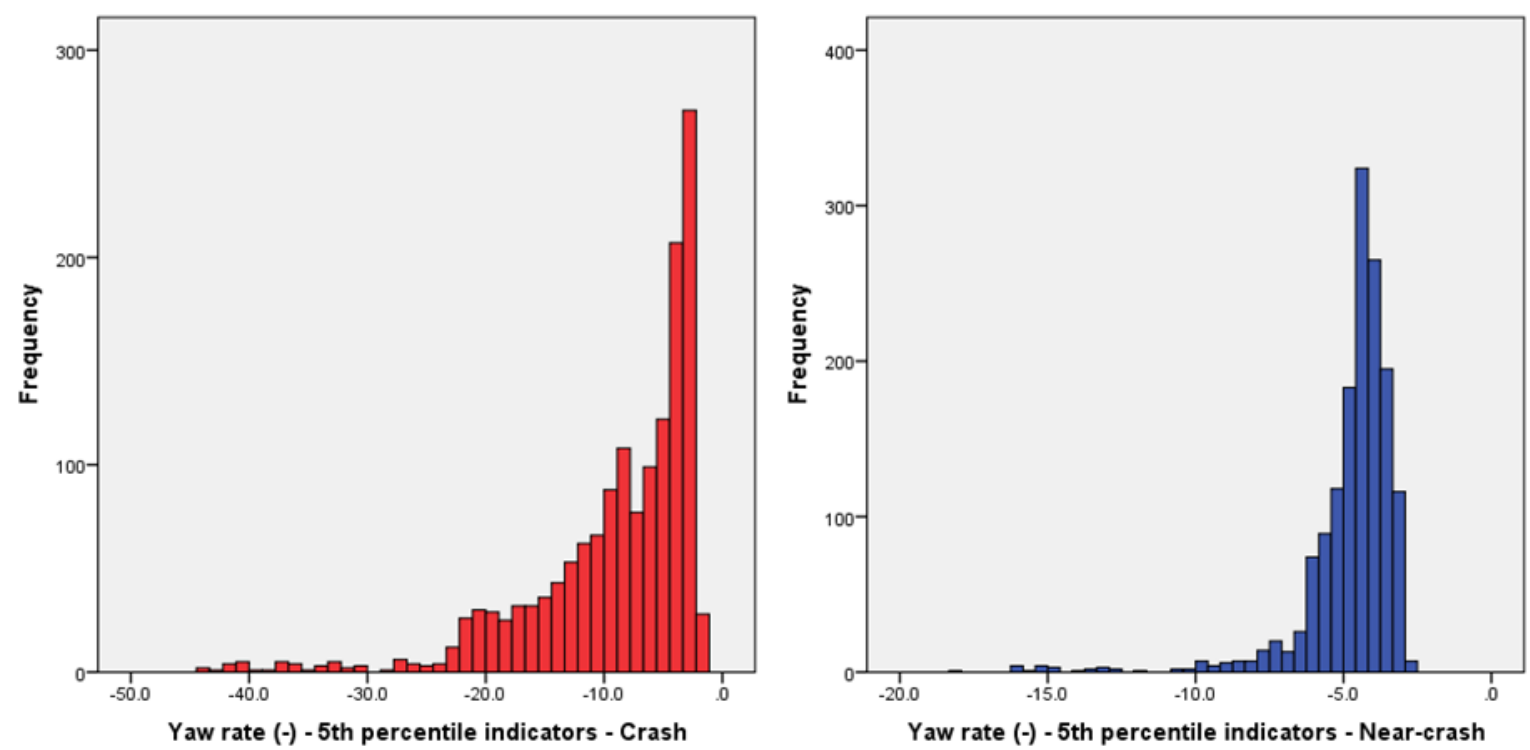

Figure 8 Distributions of Yaw rate (-) 5th percentile indicators for crash \& near crash events 


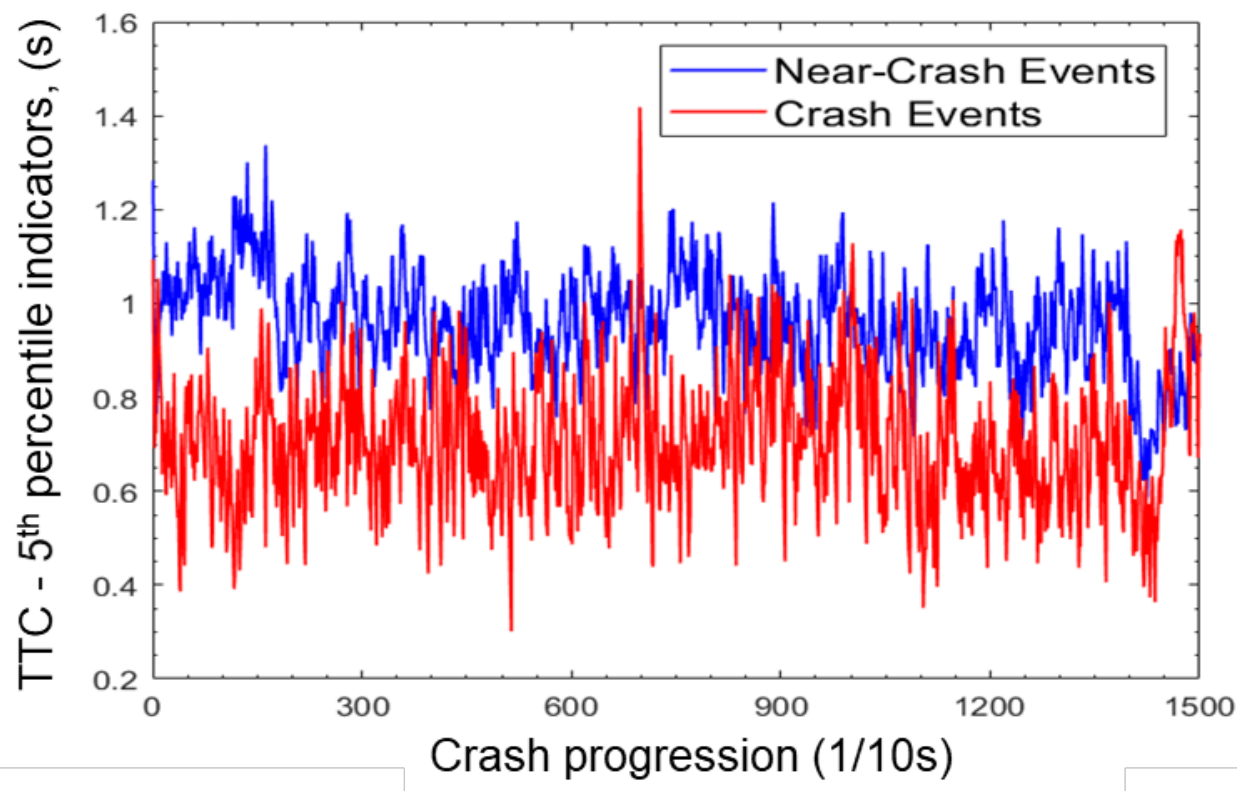

Figure 9 TTC 5th percentile indicators during crash \& near-crash development

3

Table 4 Descriptive statistics of TTC 5th percentile indicators

\begin{tabular}{lll}
\hline TTC (s) & Crashes (127) & Near Crashes (647) \\
\hline Mean & 0.707 & 0.959 \\
Median & 0.696 & 0.963 \\
Minimum & 0.301 & 0.584 \\
Maximum & 1.419 & 1.337 \\
\hline
\end{tabular}

5
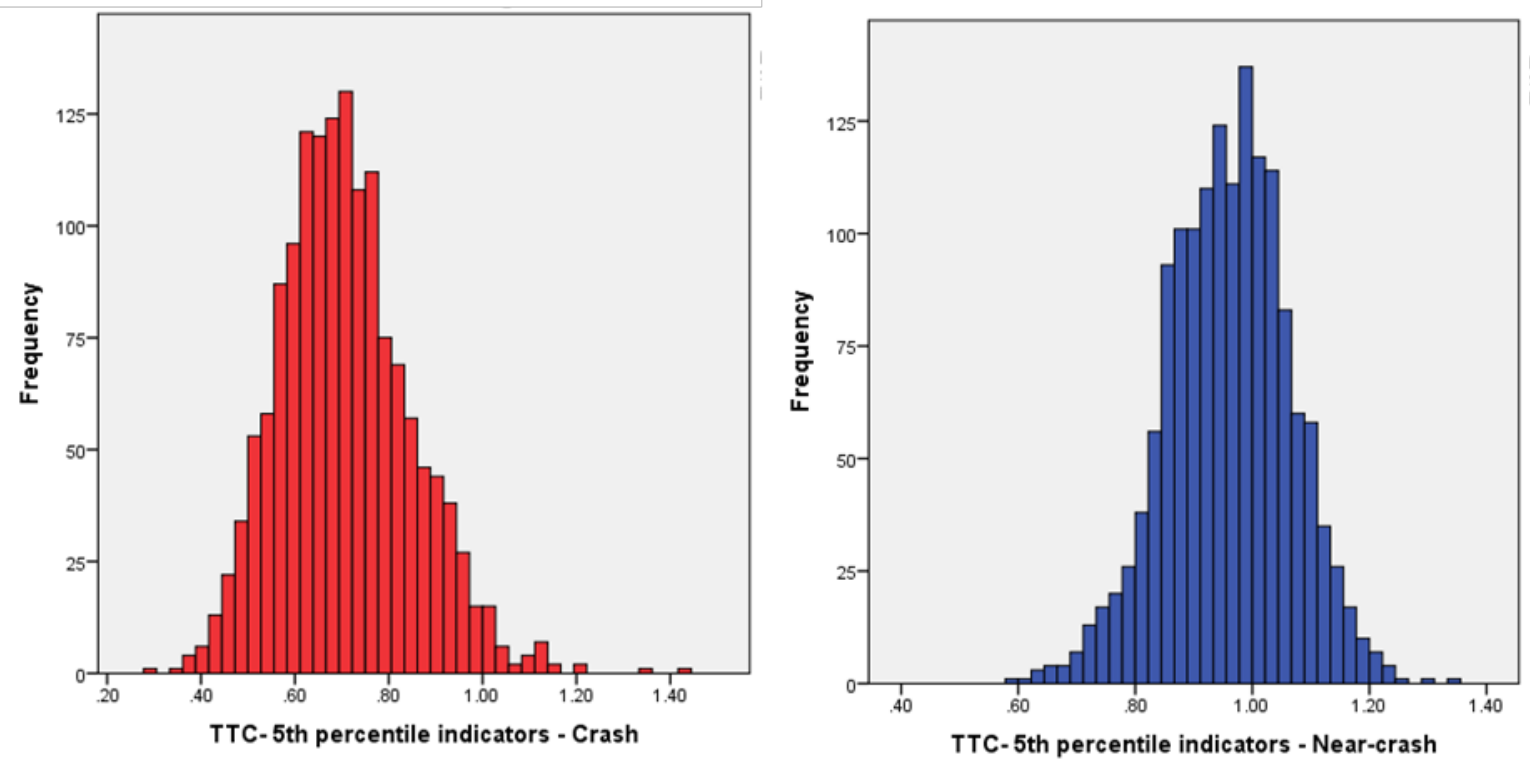

6

Figure 10 Distributions of TTC 5th percentile indicators for crash \& near crash events 
Examining the vehicle kinematics before the event revealed that an early indication of deviation could be detectable using the indicators of deceleration, lateral acceleration and yaw rate, but TTC does not follow any explicit pattern. Thus, investigating further this crash proximity indicator, and how its values change during the crash development stages will provide valuable insights in comprehension of crash mechanisms and evolution of crash risk.

\section{Analysing factors affecting TTC}

TTC values are analysed by using mixed effects hierarchical regression models. The predictor variables are gender, age, vehicle type, driven miles per year, longitudinal acceleration (both negative and positive values), lateral acceleration, yaw rate, speed, and time defined as the system generated timestamp and its quadratic term, timestamp squared, variable that was derived later for analysis purposes. This additional transformation constitutes an important choice as the aim is to investigate the evolution of TTC values within the crash or near crash development and detect any earlier change to them that could be associated with the event. Thus, a non-linear relationship between TTC values and time should be also examined. Although traffic density exists as a variable in SHRP2 NDS dataset, it was not eventually considered in the model estimation as it was not provided for every data point, but only for the moment of the event.

Initially, an unconstrained (null) model was employed to check if there is a variability in the TTC values by trip. In other words, to confirm that there is a trip effect in TTC values, so the use of mixed effect model is supported against a simple regression analysis. The results indicate that there is overwhelming evidence at the 0.05 significance level, rendering this type of model appropriate for the analysis.

A random slope model was finally estimated with one explanatory variable (vehicle type) at level-2 and 4 variables (longitudinal acceleration, speed, timestamp and timestamp ${ }^{2}$ ) at level-1. The aforementioned variables proved to be affecting the TTC values in a statistically significant way. Yaw rate, lateral acceleration, age, gender and miles driven previous year were not statistically significant and they were excluded from the model. The variables were also examined to show whether the effects (slope coefficient) varied across the trip-files. The variance associated with the slope coefficient of longitudinal acceleration, speed, timestamp and timestamp squared were found to be statistically significant. The results of the multilevel model are presented in Table 5.

The estimate of the residual variance represents the variability of the observations by trip around the regression lines. More specifically, if one best-fit line is drawn for a trip, measurements would randomly vary around this line with about $95 \%$ of the values falling within 120.114 (i.e. square root of 3606.85 * 2 ) of the line.

The vehicle classification proved to be significant and the Van\&minivan category has been selected as reference. It can be observed that for all the other vehicle types the TTC values are significantly higher. Regarding the vehicle kinematics variables, longitudinal acceleration and speed of the ego-vehicle have been found to be randomly distributed with their coefficients value varying by trip.

Since the variance of the speed coefficient is quite large (i.e. 0.549) in relation to the mean of the speed coefficient, there is a high possibility that some values of the coefficients would be positive. Additionally, since the speed coefficient has assumed to follow a normal distribution, the mean is -0.2305 and the variance is 0.549647 , Z-statistic can then be obtained to calculate the area under the normal curve between the mean and 0 . This is given by:

$$
Z=\frac{0+0.2305}{\sqrt{0.549647}}=0.31
$$


$Z=0.31$ represents a $12.2 \%$ of the area under the normal curve. This means that $50 \%+12.2 \%$ $=62.2 \%$ of the speed coefficients show a negative sign (i.e. they are negatively associated with TTC) and $37.8 \%$ of the coefficients exhibit a positive sign (i.e. they are positively associated with TTC). The speed of ego vehicle, therefore, has a mixed-effect on the TTC indicating that driver speeding behaviours play a key role in their TTC values.

Table 5 Multilevel mixed effects model

Dependent variable TTC

\begin{tabular}{lcc}
\hline Fixed effect & Coefficient & t-stat \\
Speed & -0.230543 & -8.450 \\
Longitudinal Acceleration & 42.8148 & 15.723 \\
Vehicle type: & & \\
Car & 31.8363 & 13.687 \\
Truck & 29.63 & 10.102 \\
SUV and Crossover & 31.6881 & 13.057 \\
VAN and minivan (reference) & & \\
Timestamp & 0.0250 & 5.268 \\
Timestamp & -0.0000238 & -7.797 \\
Intercept & 20.2940 & 8.838 \\
Random effect parameters & & \\
Variance of speed & 0.549647 & 18.35 \\
Variance of Longitudinal & 4696.0346 & 15.875 \\
Acceleration & & \\
Variance of Timestamp & 0.016768 & 19.042 \\
Variance of Timestamp & $6.879815 \mathrm{E}-9$ & 18.999 \\
Variance of Residual & 3606.8504 & 639.270 \\
Statistics & & \\
Number of observations & $1,161,584$ & \\
Number of groups & 774 &
\end{tabular}

Similarly, the variance of longitudinal acceleration coefficient is also large compared to the longitudinal acceleration slope itself. As the mean is 42.815 and the variance 4696.035 , the $z$-score is -0.63 . This represents a $26.43 \%$ of the area under the normal curve and shows that $73.6 \%$ of the longitudinal acceleration slopes affect positively the TTC values while $26.4 \%$ of them have a negative effect.

The timestamp variable that represents the time to a crash or a near crash (i.e. crash progression sequence starting 2.5 minutes before the event) has assumed to have a quadratic relationship with TTC. It was therefore hypothesized that the TTC values just before the crash would be 'low' in relation to other TTC values and Fermat's Theorem was employed to identify at which point in time the TTC values start to decline. The first time derivative of the function is: $\frac{d(T T C)}{d t}=b_{1}+2 b_{2} t$. Therefore, the possible extremum will be the point within the function where $\frac{d(T T C)}{d t}=0$, in which $b_{1}$ is the coefficient for the linear timestamp term, $b_{2}$ is the coefficient of the timestamp squared and $t$ is the timestamp. The critical timestamp is found to be $t=526$. Moreover, the second time derivative of the function is: $d^{2}(T T C) / d^{2}=2^{*} b=2^{*}(-0.0000238)<$ 
0 indicating that this timestamp value represents the maximum point of the function. In other words, at timestamp 526, the functional value changes and the TTC values start to drop. This critical timestamp value represents 1.62 minutes (on a timescale between 0 and 2.5 minutes) before the crash indicating that TTC values, on average, starts to decline about 1 minute before the crash, ceteris paribus.

\section{DISCUSSION}

This analysis revealed that speed, longitudinal acceleration, vehicle type and time within the event development are the most significant variables affecting TTC values. Surprisingly, the factors associated with the human characteristics, age and gender, were not found statistically significant, in opposition with previous research (Papazikou et al., 2017) that found differences related to TTC normal driving behaviours across gender and age group. One speculation is that the young drivers, overrepresented in the sample, could make it difficult to present effects of age. This needs further investigation in the future to check if the situation remains the same regarding the other indicators or using a bigger sample.

Speed and longitudinal acceleration have a mixed effect in TTC values with $62.2 \%$ and $26.4 \%$ of the coefficients correspondingly affecting them in a negative way. This percentage for speed (affecting negatively TTC) is remarkably high supporting studies that correlate high speed with higher crash occurrence probability (Elvik, 2005), (Aarts \& Schagen, 2006). The usefulness of the speed as predictor of collision probability is questionable though as it depends also on the road geometry. Meanwhile, the longitudinal acceleration proved to be mostly $(74.6 \%)$ positively associated with TTC values. This can be justified as higher accelerations can occur when the lead vehicle is at a long distance or absent. Sometimes though it is the driving style which affects the acceleration behaviour and correspondingly the TTC values (i.e. aggressive drivers, tailgating).

Most importantly, time within crash or near crash sequence, expressed as timestamp and timestamp squared, proved to be affecting TTC values significantly. This required further investigation to find out the point where a change in TTC values is detectable. It was found that at timestamp 526, 1.62 minutes before the event, the TTC values started to reduce. This seems to be very early in the event sequence, but there are several factors that could potentially explain it. Firstly, TTC is not a pure variable and there have been restrictions to the calculation and manipulation of the specific indicator. The TTC distribution includes some extremely high values, meaningless from the safety aspect, which have been replaced by the $95^{\text {th }}$ percentile TTC values threshold. It should be noted that the dataset still contains some really high values that could be problematic towards the modelling process. Moreover, in terms of individual traces when events are being investigated separately, low TTC events seem to happen very regularly during normal driving, rendering further interpretation of the real-world event more complex. Certainly, in-depth investigation of individual behaviours and exploration of TTC in combination with other variables as longitudinal, lateral acceleration and yaw rate can enlighten the pre-crash conditions employing, in a way, humans as sensors for risk perception. Lastly, but possibly most importantly, the traffic conditions of the relative trips that used for the model development played a significant role in the low (early) critical timestamp, but it was not possible at the moment to include this data in the model.

This brings to discussion one of the limitations of the study. The Road Infrastructure Data was not matched with the NDS, so the traffic conditions and the road geometry could not be used. Another limitation was that all the drivers considered had been involved in an event. 
However, this constitutes a general limitation of the SHRP2 NDS data, as there were no drivers with no events available.

Nevertheless, the generation of percentile indicators with an empirical method and the employment of HLM approach illustrated the suitability of SHRP2 NDS data for investigating pre-crash conditions and the conditions during the crash development chain were visualised to suggest that TTC as a calculated indicator, can be valuable to examine the crash sequence and the development of crash risk.

Although several methods (e.g. extreme value theory, cluster analysis, multivariate Poisson log-normal model) have been employed for NDS data investigation over the years (Jonasson \& Rootzén, 2014; Zheng et al., 2014; Wu et al., 2014), HLM has not been widely utilised. In this study, given the specific structure of the data, proved to be extremely useful and reliable statistical technique as it provides the opportunity to control for the thousands of observations derived from the same driver or the same trip.

Investigating the whole crash sequence, from a normal driving situation until the event, gave insights in the understanding of the relationship between vehicle kinematics indicators and crash risk and this knowledge gained can be exploited to inform existing warning systems or enhance Advanced Driver Assistance Systems (ADAS), to efficiently support the drivers and prevent more crashes. More specifically, monitoring situations where ADAS should start to operate and exploring the interaction between humans and vehicle at this timepoint, ADAS could pick up earlier deviations when there is time to react. In other words, using humans as sensors ADAS could predict and stop crashes by detecting changes of driving behaviour.

The existing systems are set to function in the last stages of the crash sequence and mainly when the driver cannot react anymore (Automated Braking, Electronic Stability Control, etc.), but what about the early indications of deviations that could possibly predict the crash? Systems that could detect these inconsistences in driving that are associated with crash risk may offer an opportunity to develop new systems that can intervene more effectively. The type and the magnitude of the intervention depends on the stage that the deviation is detected. The study revealed how early an event can be predicted and hopefully prevented using acceleration, yaw rate and TTC as indicators and set the stage for further investigation of the indicators that can be proved reliable in crash risk prediction. Future research could also consider different crash types and event severity, road geometry and traffic conditions.

\section{CONCLUSIONS}

This paper studies the vehicle kinematics during crash sequence with a focus on TTC values. Crash sequence is a complicated concept to investigate and comprehend, but this study has taken a step further in our understanding of the pre-crash conditions regarding vehicle kinematics, the feasibility of certain crash risk indicators, the factors influence TTC and the time within crash sequence in which a detectable change in the TTC pattern starts. More accurately, the percentile indicators give a picture of the situation from normal driving conditions until the event and indicate there is a detectable deviation 10 to 20 seconds before the crash or near crash. The model results reveal that vehicle type, speed of the ego vehicle, longitudinal acceleration and time within the crash sequence are variables that affect significantly TTC values and the critical timestamp where the TTC values started to drop is 1.62 minutes before the event. These findings could be useful in enhancing existing ADAS, to more effectively and timely detect and stop an early deviation before it culminates in a crash. 


\section{Acknowledgment}

We wish to express special thanks to Insurance Institute for Highway Safety (IIHS-HLDI) for their financial support with which the SHRP2 NDS data was obtained. Moreover, we wish to express thanks to Virginia Tech Transportation Institute (VTTI) for their help with the data acquisition and comprehension.

The findings and conclusion of this paper are those of the authors and do not necessarily represent the views of VTTI, the Transportation Research Board or the National Academies.

\section{REFERENCES}

1. Aarts, L. and I.V. Schagen. Driving speed and the risk of road crashes: A review. Accident Analysis \& Prevention, Vol. 38, No. 2, 2006, pp. 215-224.

2. Abdel-Aty, R.J. Cunningham, V. Gayah, L. Hsia, 2008. Dynamic variable speed limit strategies for real-time crash risk reduction on freeways. Transp. Res. Rec.: J. Transp. Res. Board, 2078 (2008), pp. 108-116, 10.3141/2078-15.

3. Af Wåhlberg, A.E. Driver Celeration Behavior and the Prediction of Traffic Accidents. International Journal of Occupational Safety and Ergonomics, Vol. 12, No. 3, 2006, pp. 281-296.

4. Archer, J. Traffic Conflict Technique, Historical to current State-of-the-Art. Stockholm, KTH, 2001.

5. Bagdadi, O., and A. Várhelyi. Development of method for detecting jerks in safety critical events. Accident Analysis and Prevention, Vol. 50, 2013, pp. 83-91.

6. Bagdadi, O., and A. Várhelyi. Jerky driving-an indicator of accident proneness? Accident Analysis and Prevention, Vol. 43, No. 4, 2011, pp. 1359-1363.

7. Benmimoun, M., F. Fahrenkrog, A. Zlocki, and L. Eckstein. 'Incident detection based on vehicle CAN-data within the large-scale field operational test "euroFOT"'. In: Proceedings of the 22nd International Technical Conference on the Enhanced Safety of Vehicles (ESV 2011), Washington, D.C, 2011.

8. Blanco, M., J. S. Hickman, R. L. Olson, J. L. Bocanegra, R. J. Hanowski, A. Nakata, M. Greening, P. Madison, G. T. Holbrook, and D. Bowman. 'Investigating critical incidents, driver restart period, sleep quantity, and crash countermeasures in commercial vehicle operations using naturalistic data collection: Final report', Federal Motor Carrier Safety Administration, Washington, D.C, 2008.

9. Brown, T.L., J.D. Lee, D.V. McGehee. Human Performance Models and Rear-End Collision Avoidance Algorithms, The journal of the Human Factors and Ergonomics Society, Vol. $43 \quad$ No. 3, 2001, pp. 462-482. DOI: https://doi.org/10.1518/001872001775898250.

10. Chapman, A.L. An Epidemiological Approach to Traffic Safety. Public Health Reports, Vol. 69, No. 8, 1954, pp.773-775.

11. Dingus, T. A., S. G. Klauer, V. L. Neale, A., Petersen, S. E., Lee, J. Sudweeks, M. A. Perez, J. Hankey, D. Ramsey, S. Gupta, C., Bucher, Z. R. Doerzaph, J. Jermeland, and R.R. Knipling. The 100-Car Naturalistic Driving Study: Phase II - Results of the 100-Car Field Experiment. Report No. DOT HS 810 593. Washington, D.C. National Highway Traffic Safety Administration, 2006. 
12. Elvik, R. Speed and Road Safety: Synthesis of Evidence from Evaluation Studies. Transportation Research Record, Vol. 1908, No. 1, 2005, pp. 59-69.

13. Evans, L. The Dramatic Failure of U.S. Safety Policy. TR News. Vol. 242, 2006, pp. 28-31.

14. Fancher, P., R. Ervin, J. Sayer, M. Hagan, S. Bogard, Z. Bareket, M. Mefford, and J. Haugen. Intelligent cruise control field operational test, Final Report, Volume I: Technical Report. University of Michigan Transportation Research Institute, Ann Arbor, MI, 1998.

15. Farah, H., and C.L. Azevedo. Safety analysis of passing maneuvers using extreme value theory. IATSS Research, Vol. 4, 2017, pp. 12-21.

16. Farah, H., S. Bekhor, and A. Polus. Risk evaluation by modelling of passing behaviour on two-lane rural highways. Accident Analysis and Prevention, Vol. 41, 2009, pp. 887894. http://dx.doi.org/ 10.1016/j.aap.2009.05.006.

17. Farid, M.N., M. Kopf, H. Bubb, A. Essaili. Methods to develop a driver observation system used in an active safety system. BMW Group, Ergonomie, München.

18. Hamdar S.H., L.Qin, and A. Talebpour. Weather and road geometry impact on longitudinal driving behavior: Exploratory analysis using an empirically supported acceleration modelling framework. Transportation Research Part C, Vol. 67, 2016, pp. 193-213.

19. Hankey, J. M., M.A. Perez, and J.A. McClafferty. Description of the SHRP 2 naturalistic database and the crash, near-crash, and baseline data sets, Task Report, Virginia Tech Transportation Institute, Blacksburg, VA, 2016.

20. Hanowski, R. J., M. Blanco, A. Nakata, J. S. Hickman, W. A. Schaudt, M. C. Fumero, R. L. Olson, J. Jermeland, M. Greening, T. Holbrook, R. R. Knipling, and P. Madison. 'The Drowsy Driver Warning System Field Operational Test: Data Collection Methods', Final Report DOT HS 810 035, 2008.

21. Hayward, J.C. Near miss determination through use of a scale of danger. Highway Research Record. Vol. 384, 1972, pp. 24-34.

22. Hegeman, G. Assisted Overtaking: an Assessment of Overtaking on Two-Lane Rural Roads, Netherlands Research School for Transport, Infrastructure, Logistics, Delft, The Netherlands, 2008.

23. Jonasson, J. K., \& H. Rootzén. Internal validation of near-crashes in naturalistic driving studies: a continuous and multivariate approach. Accident Analysis and Prevention, Vol. 62, 2014, pp.102-9.

24. Laureshyn, A., Å. Svensson, and C. Hydén. Evaluation of traffic safety, based on micro-level behavioural data: theoretical framework and first implementation. Accident Analysis and Prevention, Vol. 42, No. 6, 2010, pp. 1637-1646.

25. Lee, D. A Theory of Visual Control of Braking Based on Information About Time to Collision. Perception, Vol. 5, No. 4, 1976, pp. 437-459.

26. Lee, T., T. Kim, B. Kim, K. Yi and J. Lee. Advanced braking algorithm for robust longitudinal risk management. Journal of Automobile Engineering, Vol. 230, No. 11, 2016, pp. 1488-1503.

27. Lefèvre, S., D. Vasquez, and C. Laugier, 2014. A survey on motion prediction and risk assessment for intelligent vehicles. ROBOMECH Journal, Vol. 1, No. 1, 2014, pp. 1. http://doi.org/10.1186/s40648-014-0001-z. 
28. Lerner, N., J. Jenness, J. Singer, S. Klauer, S. Lee, M. Donath, M. Manser, and N. Ward. 'An Exploration of Vehicle-Based Monitoring of Novice Teen Drivers: Final Report', National Highway Traffic Safety Administration, Washington, D.C., DOT HS $811333,2010$.

29. Li, Y., L. Zhang and Y. Song. A Vehicular Collision Warning Algorithm Based on the Time-to-collision Estimation under Connected Environment. Presented at the 14th International Conference on Control, Automation, Robotics \& Vision, Phuket, Thailand, 2016.

30. Martens, M. and R., Brouwer. Linking Behavioural Indicators to Safety: What is Safe and What is Not? Presented at the 3rd International Conference on Road Safety and Simulation, Indianapolis, USA, 2011, pp. 1- 14.

31. McGehee, D. V., M. Raby, C. Carney, J. D. Lee, M. L. Reyes. 'Extending parental mentoring using an event-triggered video intervention in rural teen drivers', Journal of Safety Research, Vol. 38, No. 2, 2007, pp. 215-227.

32. Mclaughlin, S. B., J. M. Hankey, and T. A. Dingus. A method for evaluating collision avoidance systems using naturalistic driving data. Accident Analysis \& Prevention, Vol. 40, No. 1, 2008, pp. 8-16.

33. Niezgoda M., T. Kaminski, M. Kruszewski. Measuring driver behaviour indicators for traffic. Journal of kones powertrain and transport, Vol.19, No. 4, 2012, pp. 504-511.

34. Nishimoto, T., Y. Arai, H. Nishida, and K. Yoshimoto. Development of high performance drive-recorders for measuring accidents and near misses in the real automobile world. JSAE Review, Vol. 22, No. 3, 2001, pp. 311-317.

35. Olson, R.L., R.J. Hanowski, J.S. Hickman, and J. Bocanegra. Driver distraction in commercial vehicle operations. Report No. FMCSA-RRR-09-042. Federal Motor Carrier Safety Administration, U.S. Department of Transportation, Washington, DC, 2009.

36. Papazikou, E., M. Quddus, P. Thomas. Detecting Deviation from Normal Driving Using SHRP2 NDS Data. Presented at the $96^{\text {th }}$ Annual Meeting of the Transportation Research Board, Washington, D.C., 2017.

37. Park, M., S. Lee, M. Kim, J. Lee, K. Yi. Integrated differential braking and electric power steering control for advanced lane-change assist systems. Journal of Automobile Engineering, Vol. 229, No. 7, 2015, pp. 924-943.

38. Pilgerstorfer, M., K. Runda, C. Brandstätter, M. Christoph, S. Hakkert, T. Toledo, R. Ishaq, and M. Gatscha. 'DaCoTA - Deliverable 6.3, Report on Small Scale Naturalistic Driving Pilot', Austrian Road Safety Board, Loughborough University, UK, 2012.

39. Regan, M., T. Victor, J. Lee, and K. Young. Driver distraction: theory, effects and mitigation. Taylor and Francis, 2009.

40. Shariat-Mohaymany, A., A. Tavakoli-Kashani, H. Nosrati, A. Ranjbari. Identifying significant predictors of head-on conflicts on two-lane rural roads using inductive loop detectors data. Traffic Injury Prevention, Vol. 12, No. 6, 2011, pp. 636-641.

41. P.St-Aubin, N. Saunier, L.F. Miranda-Moreno, 2014. Road user collision prediction using motion patterns applied to surrogate safety analysis. Transportation Research Board Annual Meeting Compendium of Papers. 14-5363 (2014).

42. Sudweeks, J. Using Functional Classification to Enhance Naturalistic Driving Data Crash/Near Crash Algorithms, Final Report, Report 15-UT-030, 2015. 
43. Tarko, A.P., G. Davis, N.Saunier, T. Sayed, S. Washington. Surrogate Measures of Safety, White Paper, 2009.

44. Tingvall C., L. Eckstein and M. Hammer. Government and industry perspectives on Driver Distraction, Taylor and Francis, 2009.

45. VanArem, B. and A.P. DeVos. The Effect of a Special Lane for Intelligent Vehicles on Traffic Flows. TNO-INRO Report 1997-02a. Delft, The Netherlands, 1997.

46. Ward, J. R., G. Agamennoni, S. Worrall, A. Bender, E. Nebot. Extending Time to Collision for probabilisic reasoning in general traffic scenarios. Transportation Research Part C, Vol. 51, 2015, pp. 66-82.

47. Winsum W. V., and A. Heino. Choice of time-headway in car-following and the role of time-to-collision information in braking. Ergonomics, Vol. 39, No. 4, 1996, pp. 579-592, DOI: 10.1080/00140139608964482.

48. Winsum, W. and W. Brouwer. Time headway in car following and operational performance during unexpected braking. Perceptual and Motor Skills 84 (3 PART II), 1997, pp. 1247-1257.

49. Winsum, W. The Human Element in Car-Following Models, Transportation Research Part F, Vol. 2, No. 4, 1999, pp. 207-211.

50. Woltman H., A. Feldstain, J.C. MacKay, and M. Rocchi. An introduction to hierarchical linear modelling. Tutorials in Quantitative Methods for Psychology, Vol. 8, No. 1, 2012, pp. 52-69.

51. Wu, B., X. Zhu, J. Shen, X. Cang, L. Li. Analysis of Steering Model for Emergency Lane Change Based on the China Naturalistic Driving Data. SAE Technical Paper 2017-01-1399, 2017, doi:10.4271/2017-01-1399.

52. K.-F. Wu, P.P. Jovanis, 2013. Defining and screening crash surrogate events using naturalistic driving data. Accident Analysis and Prevention, 61 (2013), pp. 10-22.

53. Wu, K., and C. P. Thor. Method for Using Naturalistic Driving Study Data to Analyze Rear-end Crash Sequences. Presented at $94^{\text {th }}$ Annual Meeting of the Transportation Research Board, Washington, D.C., 2015.

54. Wu, K., J. Aguero-Valverde, and P.P. Jovanis. Using naturalistic driving data to explore the association between traffic safety-related events and crash risk at driver level. Accident Analysis \& Prevention, Vol. 72, 2014, pp. 210-218.

55. Zheng, L., K.Ismail, and X.Meng. Freeway safety estimation using extreme value theory approaches: A 44 comparative study. Accident Analysis \& Prevention, Vol.62, 2014, pp. 32-41. 